\title{
Effects of physiotherapy combined with sirolimus in a patient with vascular malformation: A case report
}

\author{
Türkan Akbayrak ${ }^{1}$, Ceren Orhan ${ }^{1}$, Emine Baran ${ }^{1}$, Serap Kaya ${ }^{1}$, Gürsoy Coskun ${ }^{1}$, Ali Varan ${ }^{2}$ \\ ${ }^{1}$ Department of Physiotherapy and Rehabilitation, Faculty of Health Sciences and ${ }^{2}$ Division of Pediatric Oncology, \\ Department of Pediatrics, Faculty of Medicine, Hacettepe University, Ankara, Turkey: \\ E-mail: takbayrak@yahoo.com
}

Received: 22 February 2016, Revised: 29 April 2016, Accepted: 13 May 2016

\begin{abstract}
SUMMARY: Akbayrak T, Orhan C, Baran E, Kaya S, Coşkun G, Varan A. Effects of physiotherapy combined with sirolimus in a patient with vascular malformation: a case report. Turk J Pediatr 2016; 58: 203-207.

The aim of the present case report was to investigate the effects of a physiotherapy program combined with sirolimus in a child patient with upper extremity edema and joint limitation due to low-flow vascular malformation. This case report included an 11-year-old male patient $(26 \mathrm{~kg}, 130 \mathrm{~cm})$ diagnosed with congenital lymphovascular malformation on the left and right chest. The patient, who had edema on the upper left extremity and experienced joint limitations, was administered complete decongestive therapy (CDT) and manual therapy in combination with sirolimus. Physiotherapy included a total of 24 sessions, 3 sessions a week for 8 weeks. Following the physiotherapy, the patient was assigned to a home therapy program, and then the maintenance phase of the CDT was initiated. Evaluations were carried out at baseline, at the end of week 8, and after 12 months. Following the physiotherapy program combined with sirolimus, a decrease in extremity volume, an increase in joint movement range, and an improvement in disease-related complaints were observed. Physiotherapy methods combined with sirolimus may be an effective treatment method in patients with vascular malformations. However, further studies with larger sample size are warranted.
\end{abstract}

Key words: vascular malformation, sirolimus, physiotherapy, complete decongestive therapy, manual therapy.

Vascular anomalies are heterogeneous group lesions and the prevalence of vascular anomalies in the general public is $10 \%^{1}$. Vascular malformations include capillary malformations, venous malformations (VMs), lymphatic malformations (LMs), venolymphatic malformations (VLMs), and various combinations of these ${ }^{2}$.

The primary treatment method that should be referred to in upper extremity vascular malformations is conservative approaches ${ }^{3}$. Hammil et al. ${ }^{4}$ showed that the use of the mTOR (mammalian target of sirolimus) inhibitor sirolimus, with its tolerable side effects, significantly improved clinical conditions of the patients. In this study, it is also showed that sirolimus is effective and reliable in the treatment of life-threatening vascular anomalies, and it should be used as an important treatment approach ${ }^{5}$.
Surgical excision and medical therapies have limited success in these patients ${ }^{6}$. The only radical treatment of vascular malformations is total surgical excision. However, with widespread malformations involving adjacent tissues and organs, it is quite challenging? ${ }^{7}$.

The primary initiative to be referred to in treatment should be a conservative treatment targeting the edema and pain ${ }^{3}$. Complete decongestive physiotherapy (CDT), which is the most common and the most effective treatment method in lymphedema, is a multicomponent approach aiming to decrease the extremity volume, maintain the skin health, and support the structures ${ }^{5}$. Manual therapy is used in physiotherapy for pain and joint limitations ${ }^{8}$.

The patient included in this case report suffered an extensive lymphovascular-malformation (LFVM); therefore, surgical excision could not 
be applied and embolization and sclerotherapy were not successful. To the best of our knowledge, there is no study in the literature investigating the effects of a physiotherapy program involving CDT and manual therapy combined with sirolimus on patients with VLMs. The purpose of the present case report was to investigate the effects of a physiotherapy program combined with sirolimus in a child patient with VLM-related edema and joint limitations.

\section{Case Report}

Patient History: Physical and demographic features and detailed medical history of an 11-year-old male patient diagnosed with VLM on the left arm and thorax were recorded. The patient was born, as a twin. The family visited hospital on third postnatal day due to the edema on the left lateral thorax on the upper left extremity of the baby. In 2010, during the administration of ultrasound guided sclerotherapy in the patient diagnosed with low-flow VLM, the fluid secreting from the lacunas in the left hand second finger was all lymphatic and the lacunas were found to be independent lymphatic components. Therefore, sclerotherapy was stopped considering that it was not helpful for the patient. The patient with a congenital vascular malformation involving the entire upper left extremity at the age of 10 was referred to the Pediatric Oncology Unit for medical treatment and to the Physiotherapy and Rehabilitation Unit for physiotherapy assessment when he was 11 .

Assessment of the patient: On inspection, skin was dry, pale red. There were many black cancerogenic-looking lumps on the skin
(Fig. 1). In palpation, the temperature of both extremities were normal. Strong thumb pressure on the involved hand left a godet (Phase 2 clinical symptom of lymphedema). Furthermore, scleroses were observed on the involved side hand indicating fibrous tissue proliferation. For disease-related complaints, a $10-\mathrm{cm}$ visual analog scale ${ }^{9}$ was used to assess the pain, discomfort-heaviness and tension experienced by the patient. Compared to the healthy side, only the wrist and elbow movement ranges of the involved side were limited. Before and after treatment, joint movement ranges were measured bilaterally using a goniometer. Joint movement ranges were normal on the healthy side ${ }^{10}$. The intensity of VLM-related lymphedema was assessed by volumetric measurement before and after treatment. While in sitting position, the patient was asked to merge his upper extremity up to the axilla level in a container topped with water at room temperature. By measuring the overflowing water amount, the extremity's volume was obtained ${ }^{11}$.

Treatment plan: In 2012, sirolimus treatment was started $1.6 \mathrm{mg}, 2 /$ day, in two divided doses at the Division of Pediatric Oncology, Department of Pediatrics at the local university hospital. In the meanwhile, CDT was administered by a physiotherapist 3 times a week for 8 weeks, a total of 24 sessions. At the beginning of the treatment, the patient first put on a compression garment to control the thoracic edema. For the isolated bump on the left thorax, a niche was made on the compression garment and a pad was placed in this niche for additional pressure. Each session took about an hour. CDT included skin-care, manual lymphatic

Table I. Left Upper Extremity Elbow and Wrist Active and Passive Joint Movement Range in Pretreatment, Post-treatment and 1-Year Follow Up Data

\begin{tabular}{lcccc} 
& \multicolumn{1}{c}{ Flexion } & Extension & Ulnar deviation & Radial deviation \\
Measurement & Pre - Post treatment & Pre - Post treatment \\
parameters & 1 Year Follow Up & 1 Year Follow Up & $\begin{array}{c}\text { Pre }- \text { Post } \\
\text { treatment }-\end{array}$ & $\begin{array}{c}\text { Pre }- \text { Post } \\
\text { treatment }- \\
\text { Up }\end{array}$ \\
\hline Elbow & $100^{\circ}-110^{\circ}-110^{\circ}$ & $105^{\circ}-112^{\circ}-114^{\circ}$ & - & 1 Year Follow Up \\
Wrist & $75^{\circ}-82^{\circ}-85^{\circ}$ & $50^{\circ}-60^{\circ}-65^{\circ}$ & $30^{\circ}-32^{\circ}-35^{\circ}$ & $20^{\circ}-20^{\circ}-20^{\circ}$ \\
\hline
\end{tabular}


drainage, low-pressure bandage application, and exercises $^{12}$ (Fig. 2). Exercises in the pattern of proprioceptive neuromuscular facilitation (PNF), pumping, mild stretching, and the normal joint movements of the shoulder, elbow and wrist, and postural balance exercises were recommended to the patient. The patient was asked to carry out the exercises at home 3 times a day, with 10 repetitions of each exercise. The patient was instructed to perform the exercises gently and slowly. To improve the limitations on the involved elbow and wrist, stretching exercises and joint and soft tissue mobilization exercises $^{8}$ (30 min) were administered by a physiotherapist 3 times a week for 8 weeks, a total of 24 sessions. After 8 weeks, the patient was informed about joint projection principles and recommendations about daily life functionality. In other words, the patient was recommended to avoid high temperature and possible traumas, correct skin care, daily use of moisturizer ${ }^{13}$, correct body mechanics, and protection of the arm during daily life activities. The patient was recommended to perform the exercises regularly, avoid tight garments to maintain lymphatic flow, and be careful about infections. After the 8-week treatment period, the first phase of CDT was completed considering the steady state in edema reduction and patient tolerance. Then, the second, maintenance phase of CDT, including skin care, compression socks, self-drainage, and exercises ${ }^{9}$, was initiated. In addition to thoracic compression garment, special compression socks for the hand and arm regions separately were prescribed (Fig. 3). The patient was followed up for 1 year after treatment.

After the treatment and in one-year-follow up, the volume of the involved extremity decreased (pretreatment-posttreatment-1 year follow up volumes for left hand: 1,750-1,250-1,350 $\mathrm{ml}$, respectively; for right hand $820-830-880$ $\mathrm{ml}$, respectively). The color of the skin was transformed from pale red to vivid pink/red. The large bumps with a cancerogenic-look shrunk, and the small bumps disappeared (Fig. 4). The temperature of both extremities were normal. Scleroses disappeared, and the skin became soft. Mild thump pressure created pitting on the involved hand (clinical phase 1 symptom), which is an indication of clinical improvement. Before and after treatment, and 1-year follow up data showed improvement in the VAS score (pretreatment-posttreatment-1 year follow up VAS scores: 8.1, 4.2, and $5.3 \mathrm{~cm}$, respectively). Slight improvement was observed in elbow and wrist active and passive normal joint movements (Table I). Substantial reduction in the volume of the involved extremity following the treatment was observed.

In the other words, one-year follow up data showed that the patient maintained the endof-treatment condition in all parameters investigated.

\section{Discussion}

Although a substantial decrease was observed in the extremity volume after the treatment and at the end of 1-year follow up, it was not similar to the volume of the other extremity. However the growth of VLMs were tried to be decelerated by drug therapy, they continued to grow and the edema was only maintained. In other words, the growth was slowed down. Bandage application decreased the lymphedema volume resulting in lowered heaviness of the extremity.

The initiatives regarding the joint motion range of the patient did not provide results close to the values of the healthy extremity. Due to disuse and immobility resulting from the congenital condition, the joints were stiff. Besides, the soft tissue volume restrained the joint movement range, which was one of the factors adversely affecting treatment that could not be improved. In order to avoid a possible traumatic effect, aggressive approaches were abstained. Manual therapy was exercised within the toleration limits of the patient.

In the literature sirolimus is used in VM and LM cases not responding well to sclerotherapy, embolization, and surgical excision. For complex lesions, multiple therapies and interdisciplinary teams are needed ${ }^{14}$. In the present case, with the start of sirolimus treatment, improvements in bumps, fissures, and bleeding on the skin were observed.

The present case report is such that it does not present the effect of either of the treatment, i.e. sirolimus and physiotherapy (CDT and manual therapy), independently, so it does not present which one of them is more effective than the other. However, there is 


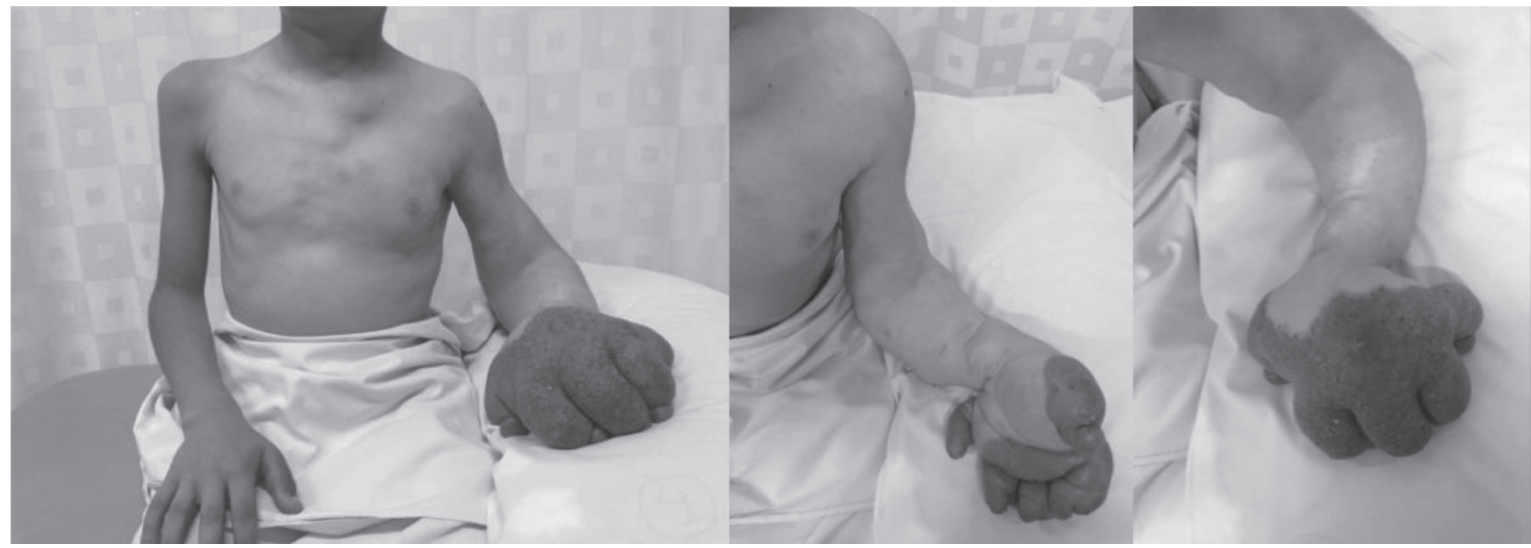

Fig. 1. Pre-treatment images

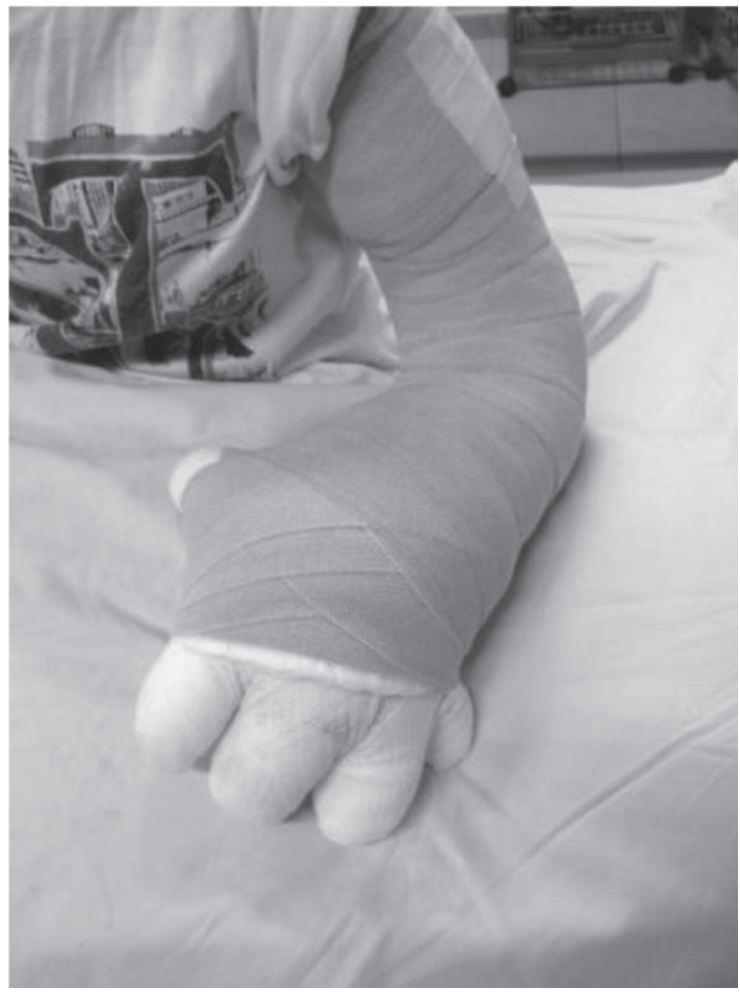

Fig. 2. Anterior aspect of CDP bandage treatment

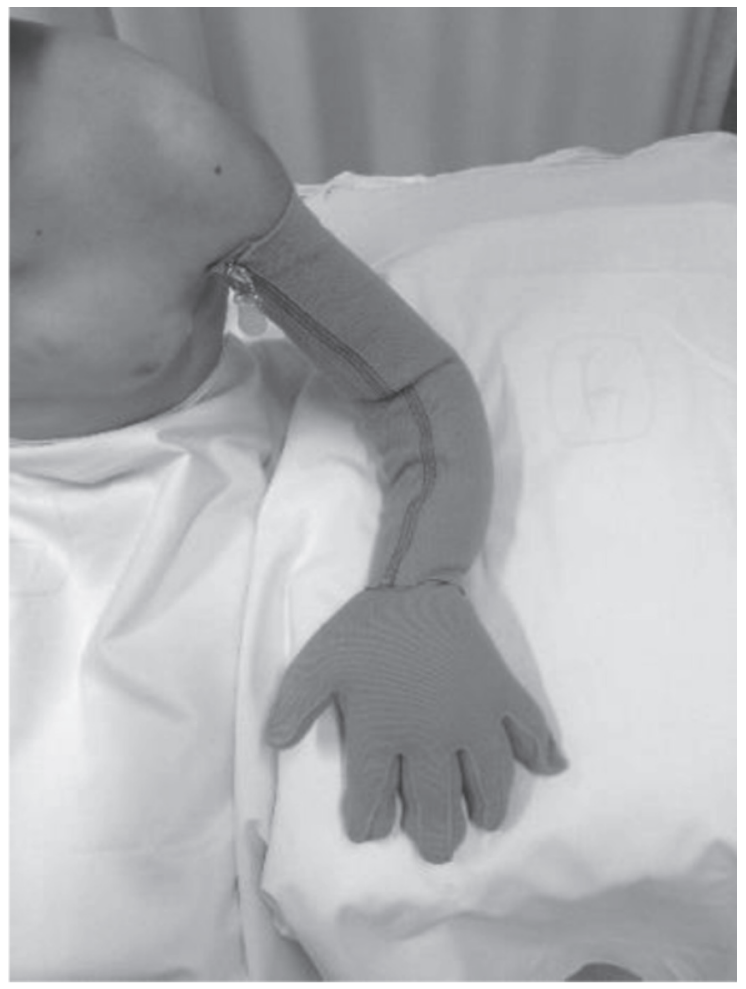

Fig. 3. Anterior aspect of the patient with planted special compression stockings
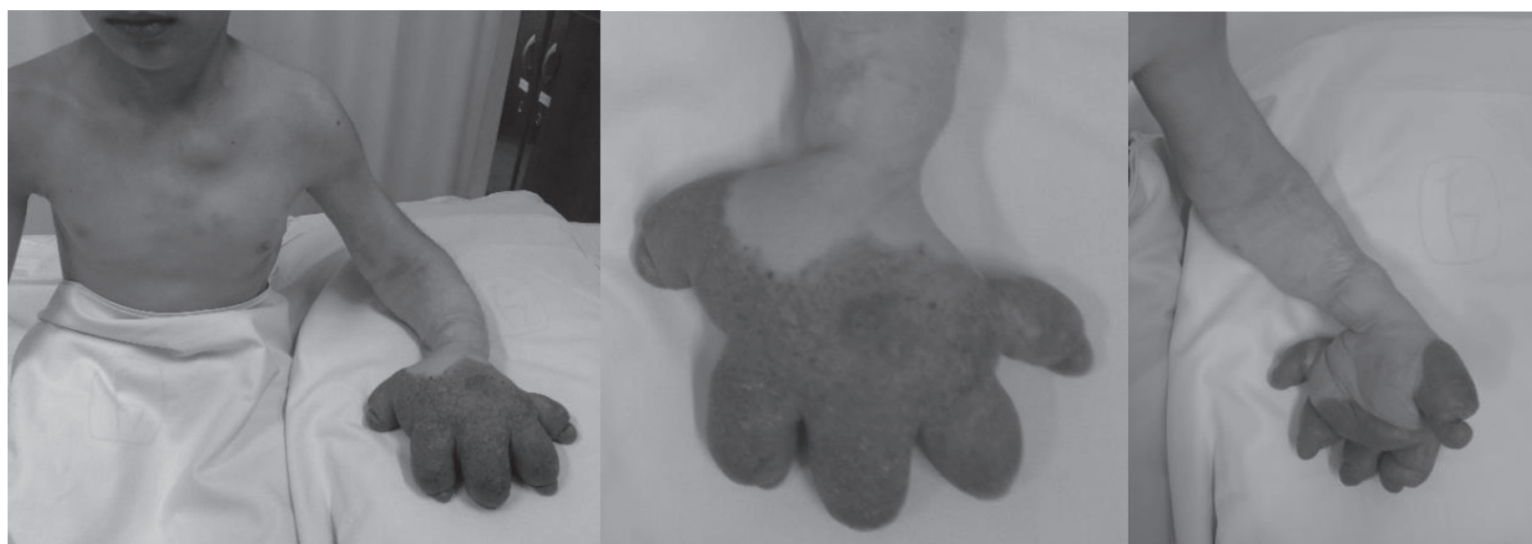

Fig. 4. Eight-week post-treatment appearance 
limited evidence in the literature regarding sirolimus treatment, and CDT is the most effective and common treatment method used in lymphedema. Therefore, we believe that the combined effects of both treatment methods yielded the effectiveness.

The present case report is important because there exists no data in the literature combining sirolimus with physiotherapy (CDT and manual therapy) and for the first time, patient history and treatment is provided in detail. Patients with complex vascular malformations, just like the present case, need multi-component evaluations and treatments.

\section{REFERENCES}

1. Blatt J, Powell CM, Burkhart CN, Stavas J, Aylsworth AS. Genetics of hemangiomas, vascular malformations, and primary lymphedema. J Pediatr Hematol Oncol 2014; 36: 5-93.

2. Akita S, Houbara S, Hirano A. Management of vascular malformations. Plast Reconstr Surg Glob Open 2014; 2: e128.

3. Ek ET, Suh N, Carlson MG. Vascular anomalies of the hand and wrist. J Am Acad Orthop Surg 2014; 22: 3-60.

4. Hammill AM, Wentzel M, Gupta A, et al. Sirolimus for the treatment of complicated vascular anomalies in children. Pediatr Blood Cancer 2011; 57: 10-24.

5. Rockson SG. Diagnosis and management of lymphatic vascular disease. J Am Coll Cardiol 2008; 52: 799-806.
6. Mohan AT, Adams S, Adams K, Hudson DA Intralesional bleomycin injection in management of low flow vascular malformations in children. J Plast Surg Hand Surg 2015; 49: 116-120.

7. Yun W-S, Kim D-I, Rho Y-N, et al. Natural course of venous malformation after conservative treatment. Surg Today 2012; 42: 950-955.

8. Kesson M, Atkins E. Orthopaedic medicine: a practical approach: Butterworth-Heinemann Medical; 1998.

9. Kaya S, Akbayrak T, Bakar Y, Topuz S. Effects of complex decongestive physiotherapy on an older female patient with psoriasis-related primary lymphedema. Top Geriatr Rehabil 2010; 26: 171-175.

10. Cole TM, Barry DT, Tobis JS. Measurement of musculoskeletal function. In: Kottke FJ, Lehman JF (ed). Krusen's Handbook of Physical Medicine and Rehabilitation (4th ed). Philadelphia: Saunders; 1990: 20-71.

11. Megens AM, Harris SR, Kim-Sing C, McKenzie DC. Measurement of upper extremity volume in women after axillary dissection for breast cancer. Arch Phys Med Rehabil 2001; 82: 1639-1644.

12. Vignes S, Blanchard M, Arrault M, Porcher R. Intensive complete decongestive physiotherapy for cancer-related upper-limb lymphedema: 11 days achieved greater volume reduction than 4. Gynecol Oncol. 2013; 131 : $127-130$.

13. Lee JH, Shin BW, Jeong HJ, Kim GC, Kim DK, Sim YJ. Ultrasonographic evaluation of therapeutic effects of complex decongestive therapy in breast cancer-related lymphedema. Ann Rehabil Med. 2013; 37: 683-689.

14. Azizkhan RG. Complex vascular anomalies. Pediatr Surg Int 2013; 29: 1023-1038. 